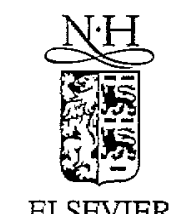

Journal of Non-Crystalline Solids 205-207 (1996) 855-860

\title{
Single particle motion in liquid sodium: a mode-coupling analysis
}

\author{
G. Nowotny, G. Kahl * \\ Institut fir Theoretische Physik, TU Wien, Wiedner Hauptstra Be 8-10, A-1040 Vienna, Austria
}

\begin{abstract}
The velocity autocorrelation function obtained in a computer experiment was interpreted in terms of a mode-coupling (MC) theory proposed by Sjögren and co-workers. The system chosen is liquid sodium at two different temperatures, 403 and $1003 \mathrm{~K}$. The MC terms of the velocity-autocorrelation function were separated into density-density, density-current and current-current contributions. For the 'binary' contribution a simple model is used; it is found that for the low temperature this functional form (with one adjustable parameter) gives reliable and satisfactory results, while for higher temperatures the model is no longer applicable. Hence, for a comprehensive application of MC theory, a better understanding of the binary effects is required.
\end{abstract}

\section{Introduction}

The interpretation of dynamic processes of simple liquids obtained from computer experiments in terms of simple hydrodynamic or memory function (MF) models [1] has reached its limits [2]: several phenomena, such as, for example, the positive dispersion for low temperatures [3] or the full-width at half maximum of the self dynamic structure factor [2] cannot be interpreted in terms of such simple models. In an effort to describe these processes, mode-coupling (MC) (or 'first principles') theories were developed about twenty years ago [4,5]. In thcir rathcr complex formalism they describe the MF of one dynamic correlation function $(\mathrm{CF})$ in terms of the other CFs, hence the name 'mode-coupling'. Computer experiments offer an ideal possibility to investigate the reliability of such methods: in contrast to most neutron scattering experiments they make the full set of CFs (i.e., single particle and collective correlation functions $\mathrm{CFs}$ ) available. The aim of this paper is to interpreted of the velocity autocorrelation function (VAC) obtained in computer experiments in terms of a $\mathrm{MC}$ theory proposed by Sjögren and co-workers [4] and to discuss the effects of temperature. To this end we have investigated two states of liquid Na. Similar studies have been performed for other CFs [6-8].

\footnotetext{
* Corresponding author. Tel.: +43-1 5880 15678; fax: +43-1 586 7760; e-mail: gkah1@tph.tuwien.ac.at.
} 


\section{Calculations}

\subsection{Basic formalism}

The basic quantity of interest is the normalized VAC $\psi(t)$ which satisfies the memory equation

$$
\dot{\psi}(t)=-\int_{0}^{t} K\left(t^{\prime}\right) \psi\left(t-t^{\prime}\right) \mathrm{d} t^{\prime}
$$

In Laplace space (characterized by argument $z$ ) an approximate expression [4] can be derived by summing repeated collisions to all orders of $K(z)$ :

$$
K(z)=\frac{K_{\mathrm{B}}(z)+R_{00}(z)+K_{\mathrm{B}}(z) R_{01}(z)}{1-R_{01}(z)-K_{\mathrm{B}}(z) R_{11}(z)-\left[K_{\mathrm{B}}(z)+R_{00}(z)+K_{\mathrm{B}}(z) R_{01}(z)\right] R_{22}(z)} .
$$

$K_{\mathrm{B}}(z)$, a rapidly decaying function in time, describes the uncorrelated binary collisions between the tagged particle and the surrounding particles. The $R_{i j}(z)$ are the mode-mode coupling terms: they describe the coupling of the self motion to long-time decay channels. These quantities may be formulated in terms of rather complicated expressions; for instance, the term representing the most important contribution to $K(z)$ in (2), i.e., the density-density decay channel, $R_{00}(t)$, is given by

$$
R_{00}(t)=\frac{1}{6 \pi} \frac{k_{\mathrm{B}} T}{m} \int_{0}^{\infty} \mathrm{d} q^{\prime} q^{4} \hat{c}\left(q^{\prime}\right)\left[S\left(q^{\prime}\right)-1\right]\left[F_{\mathrm{s}}\left(q^{\prime}, t\right)-F^{0}\left(q^{\prime}, t\right)\right] \frac{F\left(q^{\prime}, t\right)}{S\left(q^{\prime}\right)} .
$$

Similar expressions for the other $R_{i j}(t)$ are compiled in [4]. $S(q)$ and $\hat{c}(q)$ denote the static structure factor and the direct correlation function and $F(q, t)\left[F_{s}(q, t)\right]$ and $F^{0}(q, t)$ are the [self] intermediate scattering function and their free-particle limits. One possibility to reduce the complexity of Eq. (2) is to neglect all $R_{i j}(t)$ except for $R_{00}(t)$; we then obtain $K(t)=K_{\mathrm{B}}(t)+K_{00}(t)=K_{\mathrm{B}}(t)+K_{\mathrm{MC}}(t)$.

\subsection{The binary part $K_{B}$}

It is possible to show [4] that for small $t$ the non-binary contributions to $K(t)$ vanish as $t^{4}$ and that the small- $t$ decay of $K(t)$ is proportional to $\left(1-t^{2}\right)$; hence the small $t$ behaviour of $K(t)$ is entirely determined by the binary contribution $K_{\mathrm{B}}(t)$. The initial value $K(t=0)=K_{\mathrm{B}}(\mathrm{t}=0)=\Omega_{0}^{2}$ is given by the Einstein frequency (sum-rules)

$$
\Omega_{0}^{2}=\frac{n}{3 m} \int \mathrm{d} r g(r) \nabla^{2} v(r)
$$

expressed in terms of the interatomic potential, $v(r)$, and the pair distribution function $g(r), n$ is the number-density and $m$ is the mass of the particles of the liquid. Due to the complexity of the binary collisions one usually resorts to an ansatz for $K_{\mathrm{B}}(t)$, i.e., $K_{\mathrm{B}}(t)=\Omega_{0}^{2} \exp \left(-t^{2} / \tau_{\mathrm{B}}^{2}\right)$ where the binary time $\tau_{\mathrm{B}}$ can be interpreted as an average binary collision time. This binary time can be evaluated by a short time expansion of $K(t)$ under some approximations [1,9] which gives us a theoretical value $\tau_{B}^{\text {th }}$.

\subsection{Numerical details}

In order to obtain the full set of dynamic CFs we performed computer experiments by means of a standard microcanonical molecular dynamics (MD) simulation. The runs were performed for 2048 particle ensembles 
over 100000 time steps $\Delta t$ ( $\Delta t=1$ to $3 \mathrm{fs}$ ). Further details are given in [2]. We used effective pair potentials based on pseudopotential theory (for details cf. [10] and [2]). The systems investigated were two states of liquid $\mathrm{Na}$, characterized by temperatures $T$ and mass densities $\rho\left(403 \mathrm{~K} / 919.2 \mathrm{~kg} \mathrm{~m}^{-3}\right.$ and $\left.1003 \mathrm{~K} / 777 \mathrm{~kg} \mathrm{~m}^{-3}\right)$. They correspond to the states investigated by Morkel and co-workers [11,12] in neutron scattering experiments.

We calculated the MF $K(t)$ (i) by solving the memory Eq. (1) in Laplace-space $[z+K(z)] \psi(z)=1$ and (ii) by evaluating the full expression (2), using as an input the 'exact' dynamic CFs obtained in our computer experiment. Static properties, such as $g(r)$ and $S(q)$ were also calculated in the same MD runs.

\section{Results}

In Fig. 1 and Fig. 2 we show our results for the VAC $\psi(t)$ and its MF $K(t)$ for the two different Na-states investigated. $K(t)$ was calculated from the full cxpression (2), i.e., including all decay channels possible. The binary part $K_{\mathrm{B}}(t)$ was obtained using the exponential ansatz mentioned in Section 2.2.: the binary collision time $\tau_{\mathrm{B}}$ was either calculated from the approximate expression [1,9] or fitted to the small- $t$ range of the VAC (see discussion above).

The lower temperature state is a typical case for the 'cage effect': there the tagged particle is - due to the high density - enclosed in cages formed by the surrounding particles. Hence this particle will have frequent collisions with other particles: this causes a fast decay and a long-range oscillation in the VAC. Results are
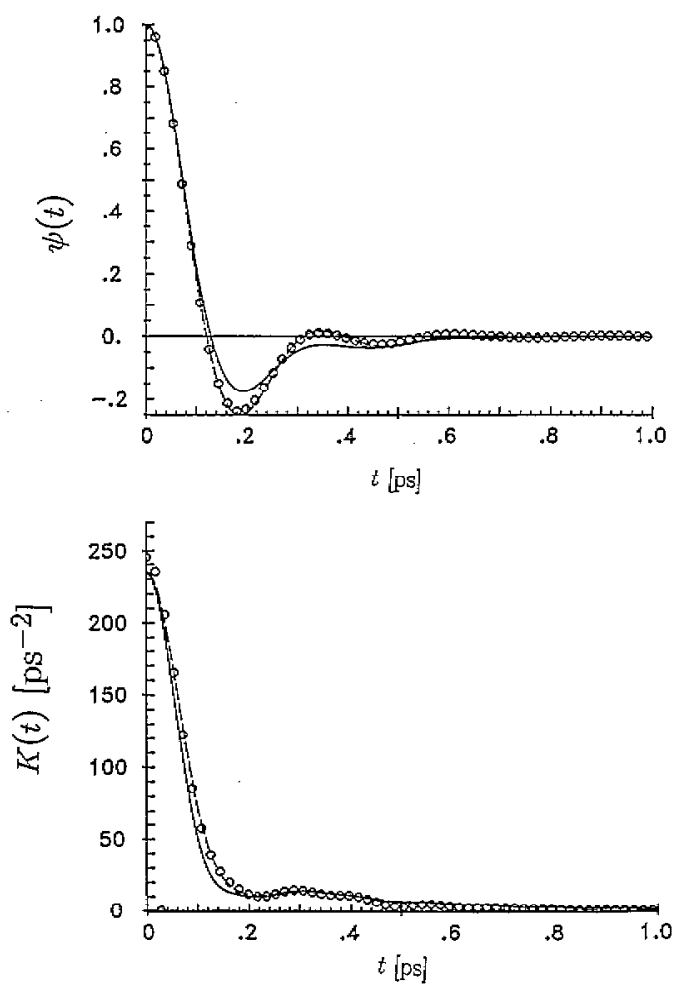

Fig. 1. Velocity autocorrelation function $\psi(t)$ (top) and its MF $K(t)$ (obtained via (2); bottom) for $T=403 \mathrm{~K}$ as functions of $t$. Symbols: full line: $K_{\mathrm{B}}(t)$ with $\tau_{\mathrm{B}}^{\text {th }}=0.069 \mathrm{ps}$; broken line: $K_{\mathrm{B}}(t)$ with $\tau_{\mathrm{B}}^{\text {fit }}=0.089 \mathrm{ps} ; \mathrm{O}=$ results from MD simulation. 

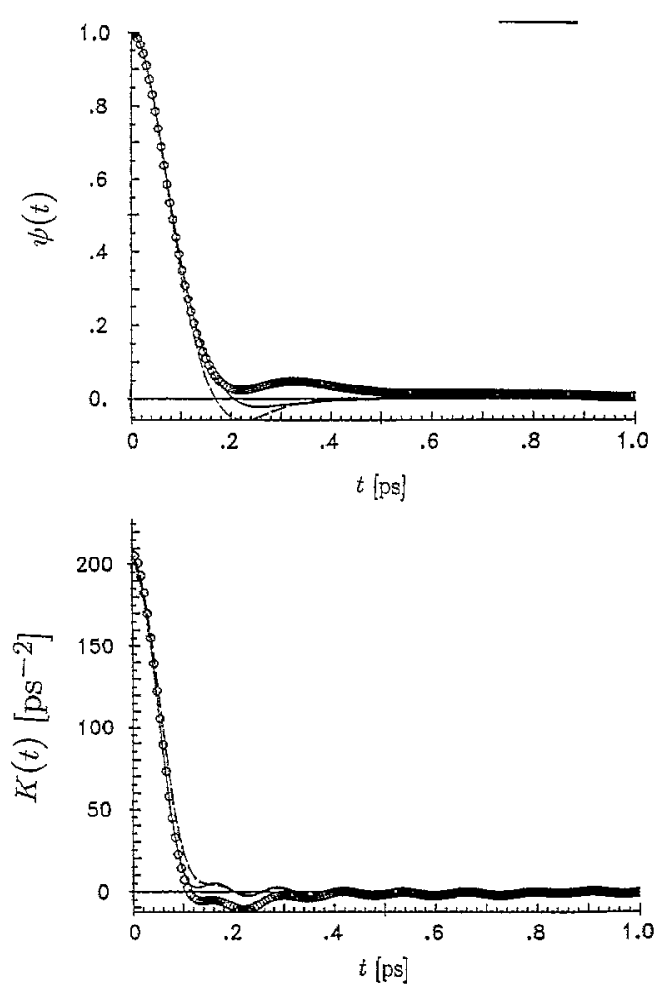

Fig. 2. Velocity autocorrelation function $\psi(t)$ (top) and its $\operatorname{MF} K(t)$ (obtained via (2); bottom) for $T=1003 \mathrm{~K}$ as functions of $t$. Symbols: full line: $K_{\mathrm{B}}(t)$ with $\tau_{\mathrm{B}}^{\text {th }}=0.061 \mathrm{ps}$; broken line: $K_{\mathrm{B}}(t)$ with $\tau_{\mathrm{B}}^{\text {fit }}=0.069 \mathrm{ps} ; O=$ results from MD simulation.

displayed in Fig. 1: for the theoretical value of the collision time $\tau_{\mathrm{B}}^{\text {th }}$ we obtained $0.079 \mathrm{ps}$ and the fitted value $\tau_{\mathrm{B}}^{\mathrm{fit}}$ is $0.089 \mathrm{ps}$. While for the first choice of $\tau_{\mathrm{B}}$ substantial differences were encountered near the first minimum in the VAC, we obtained extremely good agreement for the fitted value. We can therefore conclude that for low temperatures the simple exponential ansatz is in principle adequate for the binary collision term; however, for a reliable description a somewhat more sophisticated theory would be required to calculate the binary collision time $\tau_{\mathrm{B}}$. Small differences for the $(t=0)$ value of the MF between the model and the numerical solution of the memory equation (i.e., a violation of the sum-rule) may be attributed to numerical errors.

The other system is a typical case of the 'drift effect'. Compared to the low temperature case the particles can now move freely, hence the VAC will decay slowly in time $\left(\psi(t) \sim t^{-3 / 2}\right)$. The results displayed in Fig. 2 show that neither the theoretical value $(0.061 \mathrm{ps})$ nor the fitted value $(0.069 \mathrm{ps})$ are able to describe qualitatively the values of the $K(t)$ obtained from a numerical solution of the memory equation. Hence these discrepancies must be due to the inadequacy of the model, showing that in the expanded region we have to go beyond the simple ansatz noted above. Obviously in systems with rather low densities, the uncorrelated binary collisions follow a more complex law than given by the simple exponential ansatz. Similar results have been observed in preliminary calculations for the collective CFs; to be published in due course.

Fig. 3 finally displays the different contributions to the MF Eq. (2) for the two temperature states: for $403 \mathrm{~K}$ we see the fast decay of the binary part $K_{\mathrm{B}}(t)$ and the long time tail of the (approximated) MC term $K_{\mathrm{MC}}(t) \sim R_{00}(t)$. The contribution of the transverse channel $R_{22}(t)$ is negligible for this state (and hence not displayed). The contributions from $R_{01}(t)$ and $R_{11}(t)$ are of opposite sign and similar size and cancel to a large 

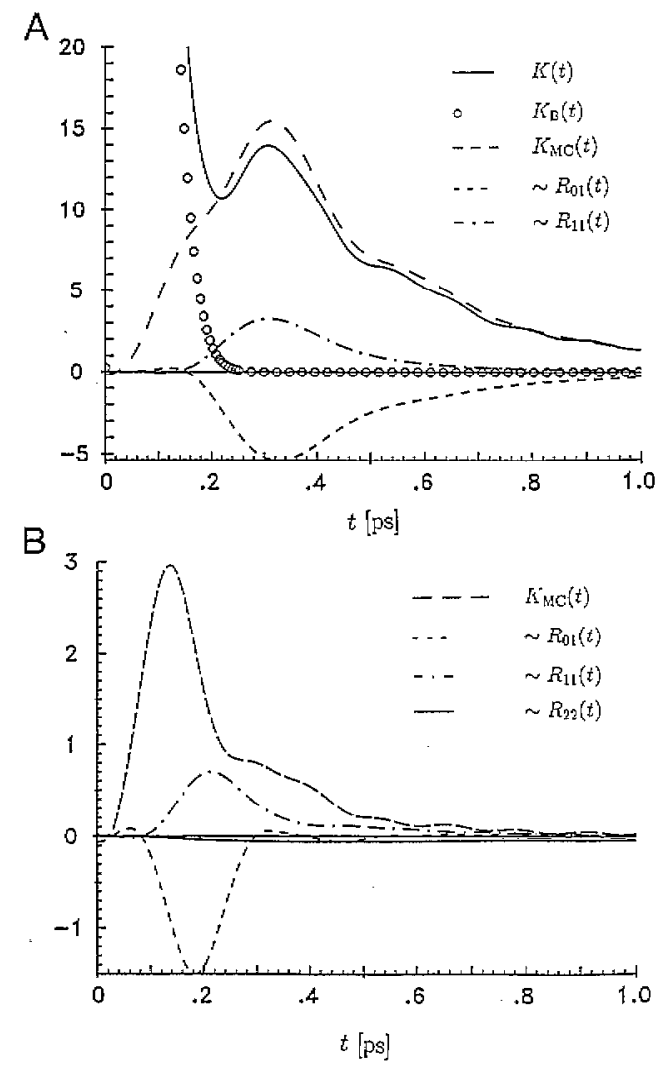

Fig. 3. MF $K(t)$, its binary part $K_{\mathrm{B}}(t)$, and the following mode-coupling terms as functions of $t$ as listed in the inset (all in [ps $\left.{ }^{-2}\right]$ ): density-density coupling $K_{\mathrm{MC}}(t)$, and the mode-coupling terms proportional to $R_{01}(t), R_{11}(t)$ and $R_{22}(t)$; (a) $403 \mathrm{~K}$ and (b) $1003 \mathrm{~K}$.

extent (which is in agreement with the results found by Sjögren [4]). Similar results have also been found at a slightly different temperature by Shimojo et al. [7]. At the higher temperature the contribution of the transverse channel is still weak but no longer negligible, which indicates that the transverse contributions are sensitively temperature dependent; the contributions from $R_{11}$ and in particular of $R_{01}$ are now much more localized in time; at that temperature a slight predominance of the $R_{01}$ contribution is observed.

\section{Conclusion}

We have investigated the temperature dependence of the VAC $\psi(t)$ and its corresponding MF $K(t)$ in terms of a MC approach for a low- and a high-temperature state of liquid Na. The required dynamic correlation functions were calculated in MD computer experiments. We have found that the reliability of the description of the MF using the formalism of MC theory depends in a very sensitive way on the model used for the binary collision term: (i) for low temperatures a simple exponential ansatz is sufficient, however, for a good agreement with computer experiment a more sophisticated theory for the determination of the binary collision time would be required; (ii) for higher temperatures, the simple exponential ansatz breaks down completely: it is no longer able to describe the functional form of the MF in a qualitative way. Here a completely new model is required. Analysing the contributions of the different decay channels to the MF we find that the largest part stems from 
the density-density channel, while the two terms describing the coupling to the longitudinal current largely cancel; the transverse decay channel gives only minor contributions.

\section{Acknowledgements}

This work was supported by the Österreichische Forschungsfonds under Project No. P8912 and the Oesterreichische Nationalbank under Project No. 4649. The authors are indebted to Professor Balucani (Firenze) for many helpful discussions.

\section{References}

[1] U. Balucani and M. Zoppi, Dynamics of the Liquid State, (Oxford, 1994).

[2] S. Kambayashi and G. Kahl; Phys. Rev. A46 (1992) 3255; G. Kahl and. S. Kambayashi, J. Phys.: Condens. Matter 6 (1994) 10897.

[3] H. Enst and J.R. Dorfmann, Physica 61 (1972) 157; H. Enst and J.R. Dorfmann, J. Stat. Phys. 12 (1975) 311.

[4] L. Sjögren and A. Sjölander, J. Phys. C12 (1979) 4369; L. Sjögren, J. Phys. C13 (1980) 705; Phys. Rev. A22 (1980) 2866; A22 (1980) 2883.

[5] W. Götze and A. Zippelius, Phys. Rev. A14 (1976) 1842; I.M. de Schepper, H. van Beijren, and M.H. Ernst, Physica 75 (1974) 1; I.M. de Schepper and M.H. Ernst, Physica A98 (1979) 189.

[6] W. Gudowski, M. Dzugutov, and K.-E. Larsson, Phys. Rev. E47 (1993) 1693; W. Gudowski, M. Dzugutov and K.-E. Larsson, J. Non-Cryst. Solids $156-158$ (1993) 125.

[7] F. Shimojo, K. Hoshino and M. Watabe, J. Phys. Soc. Jpn. 63 (1994) 1821.

[8] U. Balucani, A. Torcini, A. Stangl and Chr. Morkel, these Proceedings, p. 299.

[9] U. Balucani, R. Vallauri, T. Gaskell and S.F. Duffy, J. Phys.: Condens. Matter 2 (1990) 5015.

[10] J. Halner, From Hamiltonians to Phase Diagrams (Springer, Berlin, 1987).

[11] U. Balucani, A. Torcini, A. Stangl and C. Morkel, Phys. Scr. T57, in press.

[12] A. Stangl, PhD thesis, TU München (1994) unpublished. 